\title{
The Role of Progesterone in Traumatic Brain Injury
}

\author{
Tamara R. Espinoza, MD and David W. Wright, MD \\ Department of Emergency Medicine and Division of Emergency Neurosciences, Emory University \\ School of Medicine, Atlanta, Georgia
}

WITHIN the last 20 years, a significant body of literature has advanced our understanding of the pathophysiologic mechanisms that result from traumatic brain injury (TBI). Most importantly, we now recognize that brain injuries cause both central and systemic effects. For example, a number of studies have demonstrated increased release of proinflammatory cytokines and augmented oxidative stress reactions in peripheral tissues after isolated head injury. ${ }^{1,2}$ Thus, effective treatment modalities are those that work at multiple sites and levels of injury to enhance repair and regenerative mechanisms while limiting the destructive local and systemic processes that result from TBI.

Progesterone is a steroid hormone well known for its role in the menstrual cycle. It is produced not only by the ovaries and placenta in females but also by the adrenal glands and the brain of both sexes. Its production in the brain, by oligodendrocytes and other cell types, provides clues to its critical role in neural homeostasis. ${ }^{3}$ Indeed, the 10 -fold increase of progesterone during fetal growth is thought by some experts to be primarily for neuronal development. Within the last 20 years, pre-clinical research has repeatedly shown that progesterone has potent neuroprotective properties. There is evidence that it also plays a much broader role in correcting and maintaining homeostasis after physiologic stress and injury beyond the central nervous system. ${ }^{4}$ Most significantly, it exerts its effects through multiple proteomic and receptor-mediated systems, making it more robust than other therapies attempted previously.

\section{Early experimental research in TBI}

Progesterone initially became of interest in TBI after female rats with experimentally induced brain injury were noted to have improved outcomes and better recovery when compared with their male counterparts. ${ }^{5}$ In an early study, Roof et al ${ }^{6}$ measured cerebral edema after TBI in rats under 3 hormonal conditions: adult males with low levels of estrogen and progesterone, females in proestrus with high estrogen and low progesterone levels, and pseudopregnant females (induced by stimulating the cervix of the rat) with elevated progesterone levels. All animals were euthanized 24 hours after receiving a calibrated contusion to the medial frontal cortex. The investigators found that female rats in proestrus developed significantly less cerebral edema than male rats and pseudopregnant rats, with the highest progesterone levels, had almost no significant edema.

Corresponding Author: Tamara R. Espinoza, MD, Department of Emergency Medicine, Emory University School of Medicine, FOB Suite 126, 49 Jesse Hill Jr Dr, Atlanta, GA 30303 (tamara.espinoza@ emory.edu).

The authors declare no conflicts of interest. 
Subsequent studies found that progesterone infusion after TBI resulted in reduced cerebral edema and secondary injury in both male and female rats. To further characterize progesterone's role as a therapeutic agent after TBI, Stein and colleagues ${ }^{7}$ tested the functional recovery of rats exposed to either progesterone or placebo after sustaining an experimentally induced brain injury or sham surgery. The brain-injured rats treated with progesterone performed significantly better on spatial learning tasks than the placebo group and did nearly as well as the rats in the sham surgery group that did not sustain any brain injury.

Progesterone has subsequently been shown to be an effective neuroprotectant and treatment modality in 4 separate animal species using 22 different injury models. A review of experimental TBI studies indicates that progesterone infusion results in reduced neuronal loss, enhanced remyelination, improved functional recovery, and an overall decrease in cerebral edema. ${ }^{8}$ Investigation into the cellular and biochemical pathways mediated by progesterone provides plausible explanations for its efficacy in mitigating the effects of neuronal injury.

\section{Neurobiologic effects}

Cerebral edema is responsible for much of the secondary injury seen after TBI. Thus, edema is an important target for reducing morbidity and mortality. At both the central and systemic levels, progesterone suppresses synthesis of proinflammatory cytokines such as TNF- $a$, IL-1, and IL-6, ${ }^{9-11}$ limiting inflammation, microglial activation, and further neuronal injury. There is also evidence that progesterone reduces lipid peroxidation and free radical formation and enhances scavenger mechanisms to eliminate reactive oxygen species. Progesterone also helps to stabilize the blood-brain barrier by mediating fluid and ion exchange through membrane aquaporins ${ }^{12}$ and by regulating the synthesis of $\mathrm{p}$ glycoproteins, protein transporters that help to eliminate reactive oxygen species and inflammatory mediators within the central nervous system. ${ }^{12}$ Cumulatively, these functions serve to reduce cerebral edema and enhance cell survival in the critical hours to days following TBI.

Finally, progesterone limits neuronal apoptosis (programmed cell death) through inhibition of mitochondrial-specific proapoptotic enzymes such as cytochrome c, activated caspase-3, and the Bcl-2-associated death-promoter. ${ }^{12}$ Through mitochondrial regulation and stabilization, progesterone plays an important role in the maintenance of cellular integrity and survival.

Collectively, these processes suppress the central mechanisms that result in cerebral edema and secondary injury. Through similar peripheral pathways, progesterone infusion has also been shown to limit the inflammatory response in systemic tissues such as the spleen and mesentery, contributing to the preservation of vital organ function and overall improved outcomes after TBI. 


\section{Progesterone in clinical trials}

Two independent phase II clinical trials have shown promising results for progesterone use after acute TBI. The Progesterone for Traumatic Brain Injury, Experimental Clinical Trial III (ProTECT III) was a double-blinded study that randomized 100 patients with moderate to severe brain injury (Glasgow Coma Scale [GCS] score: 4-12) to either progesterone or placebo infusion within 11 hours of their traumatic event. Because the primary aim of the study was to determine the safety of progesterone administration, there was not sufficient power to make definitive conclusions regarding the efficacy of progesterone treatment after TBI. Nonetheless, patients in the progesterone group demonstrated a 50\% reduction in 30day mortality compared with the controls. There were no serious adverse events attributed to progesterone exposure. ${ }^{13}$

Xiao and colleagues ${ }^{14}$ subsequently performed a similar randomized, double-blinded, placebo-controlled study on 149 severely brain-injured patients with a GCS score less than 8. Mortality at 6 months was $18 \%$ in the progesterone group and $32 \%$ in the placebo group. The progesterone group also demonstrated significantly higher favorable outcomes as measured by the Glasgow outcome scale compared with controls at both 3-month (47\% vs $31 \%$ ) and 6-month (58\% vs $42 \%)$ follow-up intervals.

Despite the positive outcomes in each trial, further studies are required to validate the safety and efficacy of progesterone infusion after acute TBI. In addition, given the relatively small sample size in both studies, a larger cohort is needed to establish the generalizability of study results to the larger population. Currently there are 2 ongoing phase III clinical trials addressing these concerns, ProTECT III and Study of the Neuro-protective Activity of Progesterone in Severe Traumatic Brain Injuries.

ProTECT III is a National Institute of Neurological Disorders and Stroke, National Institutes of Health-sponsored multicenter randomized placebo-controlled double-blinded clinical trial of patients with moderate to severe TBI (GCS score: 4-12). The primary outcome is a stratified dichotomy of the Glasgow outcome scale extended at 6 months. The expected enrollment is 1140 over 4 years in approximately 30 sites nationwide. The study is running through the National Institutes of Health-funded Neurological Emergencies Treatment Trials network.

The second ongoing trial, SyNAPSE (Study of the Neuroprotective Activity of Progesterone in Severe Traumatic Brain Injuries), is being conducted by BHR Pharma (Herndon, Virginia). The trial aims to enroll approximately 1200 patients with severe TBI to either 5day continuous intravenous progesterone or placebo. The primary end point is Glasgow outcome scale 6 months postinjury.

The completion of these 2 clinical trials promises to provide the critical link between exhaustive preclinical data and rigorous clinical experience. If outcomes from the phase II trials are confirmed, progesterone will be the first successful treatment modality for moderate to severe TBI. In addition, because of its pleiotropic effects, progesterone has promise for other neurologic disorders such as spinal cord injury, penetrating brain injury, stroke, and degenerative neurologic diseases. 


\section{References}

1. Shohami E, Gati I, Beit-Yannai E, et al. Closed head injury in the rat induces whole body oxidative stress: overall reducing antioxidant profile. J Neurotrauma. 1999; 16:365-376. [PubMed: 10369557]

2. Zhou ML, Zhu L, Want J, et al. The inflammation in the gut after experimental subarachnoid hemorrhage. J Surg Res. 2007; 137:103-108. [PubMed: 17069855]

3. Baulieu EE, Robel P. Neurosteroids: a new brain function? J Steroid Biochem Mol Biol. 1990; 37:395-403. [PubMed: 2257243]

4. Wigginton JG, Pepe PE, Idris AH. Rationale for routine and immediate administration of intravenous estrogen for all critically ill and injured patients. Crit Care Med. 2010; 38:S620-S629. [PubMed: 21164406]

5. Attella MJ, Nattinville A, Stein DG. Hormonal state affects recovery from frontal cortex lesions in adule female rats. Behav Neural Biol. 1987; 48:352-367. [PubMed: 3689284]

6. Roof RL, Duvdevani R, Stein DG. Gender influences outcome of brain injury: progesterone plays a protective role. Brain Res. 1993; 607:333-336. [PubMed: 8481809]

7. Roof RL, Duvdevani R, Braswell L, et al. Progesterone facilitates cognitive recovery and reduces secondary neuronal loss causes by cortical contusion injury in male rates. Exp Neurol. 1994; 129:64-69. [PubMed: 7925843]

8. Stein DG, Wright DW, Kellermann AL. Does progesterone have neuroprotective properties? Ann Emerg Med. 2008; 51:164-172. [PubMed: 17588708]

9. Chen G, Shi J, Ding Y, et al. Progesterone prevents traumatic brain injury-induced intestinal nuclear factor kappa B activation and proinflammatory cytokines expression in male rats. Mediators Inflamm. 2007; 2007:93431. [PubMed: 18274644]

10. Stein DG. Progesterone exerts neuroprotective effects after brain injury. Brain Res Rev. 2008; 57:386-397. [PubMed: 17826842]

11. Amiry-Moghaddam M, Otsuka T, Hurn PE, et al. An alpha-syntrophin-dependent pool of AQP4 in astroglial end-feet confers bidirectional water flow between blood and brain. Proc Natl Acad Sci USA. 2003; 100:2106-2111. [PubMed: 12578959]

12. Stein DG, Wright DW. Progesterone in the clinical treatment of acute traumatic brain injury. Expert Opin Investig Drugs. 2010; 19:847-857.

13. Wright DW, Kellermann AL, Hertzberg VS, et al. ProTECT: a randomized clinical trial of progesterone for acute traumatic brain injury. Ann Emerg Med. 2007; 49:391-402. [PubMed: 17011666]

14. Xiao G, Wei J, Yan W, et al. Improved outcomes from the administration of progesterone for patients with acute severe traumatic brain injury: a randomized controlled trial. Crit Care. 2008; 12:R61. [PubMed: 18447940] 